
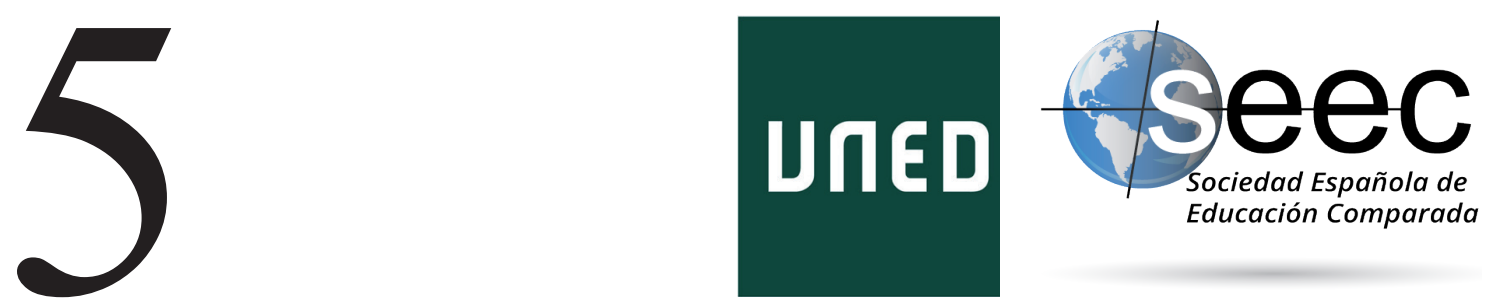

\title{
El debate Modernismo versus Postmodernismo: su impacto en la universidad
}

The debate Modernism versus

Postmodernism: impact in the university

\section{María José García Ruiz*; Sagrario Crespo Garrido**}

DOI: $10.5944 /$ reec.40.2022.31050

\author{
Recibido: 25 de junio de 2021 \\ Aceptado: 14 de septiembre de 2021
}

\footnotetext{
*María José García Ruzz: es Profesora Titular de Educación Comparada del Departamento de Historia de la Educación y Educación Comparada de la Facultad de Educación de la Universidad Nacional de Educación a Distancia (UNED). Es miembro de la Junta Directiva de la Sociedad Española de Educación Comparada, SEEC, y Secretaria de la SEEC. Es Directora de la Revista Española de Educación Comparada, REEC. Es miembro de la Comparative Education Society of Europe, CESE. Datos de contacto: E-mail: mjgarcia@edu.uned.es. **Sagrario Crespo Garrido: es Licenciada en Farmacia. Máster en Bioética. Doctora en Humanidades y Ciencias Sociales. Profesora de Bioética de la Universidad Francisco de Vitoria. Datos de contacto: E-mail: sagrario.crespo@ufv.es
} 


\title{
Resumen
}

El postmodernismo, que se sustenta epistemológicamente en el postestructuralismo, ha marcado su huella en educación con las realidades de «fragmentación», «incoherencia»y «caos político». Aunque hay académicos que aseveran que «no existe una teoría postmoderna de la educación» (Green, 1994), en la actualidad asistimos al debate de académicos modernistas versus teóricos postmodernos que reivindican, respectivamente, su visión de la universidad en términos de la idea de la universidad, la teleología, las funciones sociales y la epistemología de la institución universitaria. Especialmente imperioso se revela el análisis de las aportaciones del Proceso de Bolonia en la institución universitaria y en el debate modernismo-postmodernismo, en tanto que la potestad educativa de la Unión Europea ha dejado una huella en la tradición educativa universitaria del continente europeo. En suma, entre las conclusiones de este debate destaca la imperiosidad de potenciar las ciencias humanas en tanto que la universidad sigue ejerciendo su rol de liderazgo político y social de la sociedad occidental. A las humanidades y las ciencias sociales compete, más que a otras disciplinas actualmente promovidas por el profesionalismo imperante, el diseño del modelo de hombre y de sociedad en el siglo XXI. Sin esa orientación, la misma sociedad queda al dictado de la economía como única vigía social, con todos los riesgos, limitaciones y empobrecimiento social y moral que esto supone.

Palabras clave: modernismo; postmodernismo; universidad; epistemología; teleología; Proceso de Bolonia; sociedad occidental.

\begin{abstract}
Postmodernism, while supporting epistemologically itself in the poststructuralist theory, has marked its footprint in education with the realities of 'fragmentation', 'incoherence' and 'political chaos'. While there are academics which assert that 'there is not a postmodern theory of education' (Green, 1994), we assist currently to the debate of modern academics versus postmodern theorists that reclaim, respectively, their particular vision of the university in terms of the idea of the university, the teleology, social functions and the epistemology in the university institution. Specially imperative is the analysis of the contributions of the Bolonia Process in the university institution and in the debate modernism-postmodernism, for the educational competency of the European Union has left a footprint in the university educational tradition of the European continent. In sum, among the conclusions of this debate it is essential the promotion of the humanities, in the sense that it belongs to the university the mission of the social and political leadership of Western society. It is the responsibility of the humanities and the social sciences -more than of other practical disciplines currently promoted- the design of the model of man and society of the XXIst century. Without such orientation, the society rests in the dictate of the economy as only social lookout, with all the risks, limitations and impoverishment that this implies.
\end{abstract}

Keywords: modernism; postmodernism; university; epistemology; teleology; Bolonia Process; Western society. 


\section{Origen y significado del postmodernismo y términos afines}

Desde que Val D. Rust denunciara en su artículo de 1991 la indiferencia de la comunidad de comparatistas de la educación por el fenómeno del postmodernismo, se ha incrementado notablemente la producción académica de artículos y trabajos que operan el análisis y la reflexión del movimiento postmoderno y su impacto en la ciencia comparada de la educación y en la educación globalmente considerada (Paulston \& Liebman, 1994; Cowen, 1996; Jarvis, 1996; Young, 1997; Peters, 1998; Paulston, 1999; Mehta \& Ninnes, 2000; Ninnes \& Mehta, 2000; Ninnes \& Burnett, 2003; Cowen, 2003; Welch, 2003). Según Green (1994), el postmodernismo ha devenido central a los debates teóricos. Afirma que el discurso ha devenido un punto de referencia teórico en relación al cual tienen que posicionarse todos los productos intelectuales: «los debates en Sociología de la educación (y esto podría extenderse a todas las ciencias sociales - la apostilla es nuestra) han adoptado un creciente tono postmoderno». En su didáctico y clarificador libro, recomendable a todos los neófitos en relación al movimiento postmoderno, Hans Bertens (1995) ubica el origen de la aparición del término postmodernidad en el seno de varios debates artísticos y culturales de la década de 1950. La misma periodicidad es propuesta por el comparatista Val D. Rust (1991, p. 610). Otros académicos, como Perry Anderson, remontan más atrás el origen de este fenómeno. Anderson afirma que la idea de postmodernismo emergió primero en el intermundo hispano en los años treinta del siglo XX, una generación antes de su aparición en Inglaterra y en los EE.UU. (Anderson, 2000, p. 8). La aparición de este término en el mundo anglófono no se produjo sino hasta veinte años más tarde, en un contexto muy distinto, y como categoría histórica más que estética. Finalmente, no son pocos los autores que ubican el origen del postmodernismo en la crítica radical de los paradigmas y valores modernos realizada por Nietzsche en el siglo XIX. Son de esta última visión académicos como el historiador Ignacio Sotelo (2003), y el sociólogo historiador británico Andy Green (1994).

El postmodernismo, también denominado post-humanismo, posthistoricismo (Kunitz, 1955) o post-Occidente (Olson, 1987), surge como reacción a la epistemología moderna y sus formulaciones universalistas, lineales, sus métodos sencillos, su postulado de la validez de relaciones causales regulares y no ambiguas entre causas y efectos; y su convicción de la existencia objetiva de la verdad y su posible conocimiento por el científico o el filósofo con los métodos adecuados (Carter, 1998, p. 7). Por el contrario, los rasgos epistemológicos característicos del conocimiento postmoderno se concretan en un amplio escepticismo epistemológico, un gran subjetivismo ético, una desconfianza manifiesta de la razón, y una sospecha del rol de la ideología en el mantenimiento del poder político y económico (García Ruiz, 2011). Entre los postulados epistemológicos postmodernos hay algunos elementos que han merecido especial atención por los académicos. Mención especial al respecto merece, muy particularmente, el relativismo epistemológico y ético, en relación al cual no pocos académicos disienten. En puridad, el relativismo epistemológico incide de lleno en la posible construcción del conocimiento en la academia, y en la validez del mismo, algo que analizaremos más adelante al abordar el impacto del paradigma postmoderno en la universidad.

El postmodernismo se vincula con ciertos fenómenos y términos adyacentes que comparten algunos rasgos con el mismo teniendo, a la vez, su propia especificidad. Algunos 
de estos términos son el postestructuralismo, el post-fordismo, el postindustrialismo, la deconstrucción, la normalización y la performatividad (Carter, 1998, pp. 9-12). De este elenco de términos sociológicos y filosóficos, el que mayor afinidad revela con el contenido del paradigma postmoderno es el del postestructuralismo. Carter (1998, p. 8) revela la deuda del postmodernismo con los influjos del postestructuralismo francés de Foucault, Derrida, Baudrillard y otros. Tal es, de hecho, la conexión del postmodernismo con el postestructuralismo, que hay académicos que manifiestan su interpretación de que el postmodernismo es, en realidad, postestructuralismo con otro nombre. Otros autores abogan por que el postestructuralismo puede ser concebido como una escuela o variante en el seno del postmodernismo (ibídem, 9), siendo esta una cuestión sujeta a debate. El movimiento del postestructuralismo que, sin duda, constituye la base y el cimiento teórico del postmodernismo, tiene, según Green (1994), una deuda con los escritos de Nietzsche, de donde derivan su énfasis en el carácter múltiple y heterogéneo de la realidad, y la naturaleza contingente e inestable del sujeto individual, por debajo de cuya aparente unidad subyace solo una confusión de conflictivos deseos y de impulsos. Lo que Nietzsche concluyó es que todo conocimiento es relativo, válido solo en el seno de su propio marco conceptual. Los postestructuralistas adoptan enteramente su relativismo.

Hay otro término ampliamente utilizado por los diversos académicos al aludir a la educación postmoderna, y es el de performatividad (Ball, 1998; Cowen, 1996). Siguiendo a Ball, puede afirmarse que la performatividad «desempeña un rol particular en la reorientación de la educación, las instituciones educativas y los estudiantes a la necesidades competitivas de la economía» (Ball, 1998, p. 189). Ball cita a Yeatman (1994, p. 111) cuando afirma que «la performatividad es un principio de gobierno que establece relaciones funcionales estrictas entre un estado y sus medios internos y externos».

Pese al desarrollo epistemológico de la teoría postmoderna, en estos años de la tercera década del siglo XXI esta postura dista de ser la mayoritaria en los debates, siendo lo más frecuente la existencia de una dicotomía modernismo versus postmodernismo y el análisis de sus implicaciones esenciales en el ámbito de la educación. No en vano académicos como Green afirman que «aún no existe algo como una teoría postmoderna de la educación» (Green, 1994, p. 68).

La incidencia de este debate del modernismo y el postmodernismo en educación tiene especial interés en la institución de la universidad, dado el actual protagonismo político y económico de la universidad en la sociedad del siglo XXI y que exponemos a continuación.

\section{La universidad occidental: nivel educativo clave en el siglo XXI}

En la actualidad, como consecuencia de la aparición de fenómenos como la globalización, de procesos como el de Bolonia, y de la argumentación de los análisis postmodernos, la universidad europea está siendo objeto de intenso escrutinio en relación a su misión en la sociedad, las funciones que desarrolla y los objetivos que pretende cumplimentar. Se trata de una postura revisionista de la concepción teleológica y epistemológica que tradicionalmente ha predominado en la universidad con relación a estas cuestiones. Adicionalmente al impacto que los fenómenos mencionados han ejercido en la institución universitaria, se pueden enumerar tres causas concretas que, en el marco de los fenómenos anteriores, explican el actual protagonismo político y económico de la universidad en 
la sociedad del siglo XXI. La primera causa tiene un carácter contextual, y se ubica en la idea defendida por muchos académicos relativa a la condición de nuestro momento histórico actual como una época de tránsito de los tiempos modernos a los postmodernos. En este sentido, algunos autores destacan que, históricamente, en momentos de emergencia de una «sociedad nueva», la universidad ha sido convocada para ayudar a diseñar y consolidar el cambio (Fernández, 1999, p. 210). Así, del mismo modo que, a comienzos del siglo XIX el Estado prusiano acudió para su proceso de regeneración a Humboldt y a la Universidad de Berlín por él creada, también a comienzos del siglo XXI, la universidad es ahora convocada por la Unión Europea y los Estados Miembros para repensar los fines y la naturaleza del conocimiento en momentos de emergencia de la actual sociedad de la información y del conocimiento. La segunda causa del actual prolijo análisis al que está siendo sometida la institución universitaria reside en el carácter mismo de los cambios económicos y sociales que están produciéndose en los primeros años del siglo XXI: como acabamos de apuntar, avanzamos hacia una sociedad del conocimiento en la que, necesariamente, la universidad constituye una de las instituciones rectoras de las transformaciones en curso, en tanto que el conocimiento es el elemento propio de análisis de la institución universitaria. Por último, destacar que la tercera causa del protagonismo de la institución universitaria en nuestros días radica en la condición instrumentalizada de que ha sido objeto la educación en numerosos momentos históricos para operar cambios sociales, y también en nuestros días: la Unión Europea ha consignado explícitamente en el Tratado de Maastricht (1997) que desea instrumentalizar la educación para desarrollar su proyecto político, económico, social y cultural europeo. Dicha instrumentalización ha comenzado por la universidad, institución tradicionalmente mentora de los niveles educativos previos (García Garrido, 1998, p. 47).

Con el objeto de valorar el impacto y el alcance del movimiento de reforma en la enseñanza superior, y de ponderar, en su justa medida, en qué grado los elementos de la tradición continúan o si, en su caso, deben permanecer vigentes, los epígrafes que siguen tienen por cometido el análisis de las transformaciones que actualmente se están operando en la enseñanza universitaria. En concreto, se exponen los debates que se están desarrollando en Europa en el momento presente entre los denominados académicos modernistas y los postmodernos. En este sentido, se ofrecen las diversas posturas que ambos grupos de estudiosos defienden con relación a cuestiones como el carácter de la institución universitaria, el concepto que los diversos académicos poseen de la universidad, su concepción teleológica de la universidad, las funciones sociales que se asignan a esta institución, y el concepto que proponen con relación a la epistemología de la institución universitaria.

\section{Transformaciones actuales en la enseñanza superior}

Hay académicos que afirman que la universidad contemporánea está en crisis (Cowen, 1996, p. 245). Podríamos afirmar, con mayor exactitud, que la universidad actual está en crisis porque lo está la sociedad en la que dicha universidad está inserta. No puede sorprender, pues, la centralidad recurrente de la temática de la institución universitaria en los diversos foros internacionales y nacionales actuales (cf. Comisión Europea, 1993; Comisión Europea, 2006; Smith y Webster, 1997). En dichos foros, dista de haber 
consenso con relación a la naturaleza y las funciones presentes y futuras de la institución universitaria. Así, aun a riesgo de simplificar, como hemos avanzado más arriba, podríamos categorizar las posturas existentes en dos extremos. Por un lado, podemos destacar la postura de aquellos académicos modernistas que defienden que la idea de la universidad europea del siglo XXI es una continuidad y evolución de la tradición liberal que contempla la adquisición del saber como un fin suficiente en sí mismo (cf. Filmer, 1997; Kumar, 1997). De hecho, este grupo de académicos cuestiona la existencia real y operante de una era "postmoderna». Así, autores como Filmer establecen que «la lógica de los posts -sociedad postmoderna, epistemología postestructuralista y economía postindustrial- es una que informa la teoría especulativa, pero no es aún una socio-lógica, por lo cual no tiene correlatos sociales o culturales sustanciales y no puede proveer, por ello, una adecuada consideración del rol social de la enseñanza superior» (Filmer, 1997, p. 57). En el otro extremo, podemos citar la visión de aquellos otros académicos postmodernos que defienden que la institución universitaria está alcanzando y se encamina hacia tal grado de diferenciación, especialización y profesionalismo, que ello exige una auténtica redefinición revolucionaria de funciones sociales, entre las que la función de innovación tendría un lugar predominante (cf. Bauman, 1997; Scott, 1997).

Más allá de las divergencias en los contenidos de sus discursos, que analizaremos más adelante, en lo que sí hay un consenso manifiesto por parte de ambos grupos de académicos es en el reconocimiento de los cambios que ha experimentado la universidad en los últimos años, y que han afectado, en parte, a la naturaleza de esta institución. Así, los cambios más relevantes que se han producido en la universidad se cifran en el tránsito de una cultura de elite en la universidad a una cultura de masas, la drástica disminución de recursos tradicionalmente destinados a esta institución, la devaluación del valor de las titulaciones universitarias (consecuencia del crecimiento en el número de estudiantes), la adopción de una gestión de tintes empresariales por la universidad, el impacto de la tecnología de la información (que posibilita la impartición de la enseñanza más allá de los confines geográficos del campus tradicionalmente concebido), y la supuesta conducción de la universidad según una agenda predeterminada por el gobierno y la industria (que condiciona una enseñanza de destrezas y una investigación acorde a los intereses gubernamentales) (Smith y Webster, 1997, pp. 1-5). Otros autores suman a estos cambios otras modificaciones cruciales en la enseñanza superior europea, como la diferenciación institucional (el desarrollo de nuevas formas de enseñanza superior que han posibilitado la expansión cuantitativa de este nivel de la enseñanza), el desarrollo de nuevas formas de enseñanza y aprendizaje, y la europeinización de la enseñanza superior (Gellert, 1993, pp. 17-19). De los cambios citados, quizá el que incide en mayor medida en el cambio de la naturaleza de las universidades es el primero, es decir, el que está provocando el tránsito de una universidad concebida para la elite social a una diseñada para las masas. Ello especialmente en el modelo anglosajón de universidad, más que en los modelos francés o alemán de universidad, debido al ideal británico de «intimidad» entre profesores y estudiantes (Gellert, 1993, pp. 17-19). Las transformaciones aludidas experimentadas por la actual universidad europea explican el cambio progresivo de funciones que está sufriendo esta institución. Son cambios que pueden alterar, a largo plazo, el enfoque funcional históricamente característico de los diversos sistemas de enseñanza superior (ie. el modelo alemán de investigación, el modelo francés profesionalizador, y el modelo inglés personalista). 


\section{Los debates entre académicos modernistas y postmodernos con relación a la universidad}

Como se ha especificado en líneas anteriores, en los primeros años del siglo XXI se está produciendo un extenso debate en el entorno académico del ámbito occidental acerca de las implicaciones de la globalización en la institución universitaria. Dicho debate se polariza en dos posturas extremas, de académicos modernistas y postmodernos, si bien hay muchos autores que sostienen posturas intermedias. A grandes rasgos, podría decirse que el efecto principal de la globalización en la universidad europea actual está radicando en el triunfo, al menos coyuntural, de la agenda postmodernista en la idea, la teleología, la concepción de las funciones sociales y la epistemología de la universidad en Europa. No obstante, no hay que desdeñar la solidez de la argumentación teórica de los académicos modernistas en su defensa de la permanencia de ciertos elementos cruciales de la institución universitaria. Resulta preciso -hecho que vamos a realizar a continuación- realizar un análisis de las concepciones de ambos grupos en ámbitos diversos de la institución universitaria como, especialmente, la idea de la universidad europea, la teleología de la universidad, las funciones sociales de la universidad, y la epistemología de la institución universitaria en Europa.

\subsection{El debate sobre la idea de la universidad europea}

Algunos académicos como Cowen, han advertido de la «confusión actual acerca de lo que sea la universidad» (Cowen, 1996, p. 245). Este académico ha puesto de manifiesto la existencia de una pluralidad de definiciones diversas, no siempre coincidentes, acerca de la entidad de la institución universitaria, como la que la define como «institución con el derecho de emitir una titulación de doctorado» (Cowen, 1996, p. 245). La confusión y disparidad de ideas acerca de la institución universitaria es real, si bien no es, propiamente, actual. Esta diversidad de concepciones viene produciéndose a lo largo de todo el siglo XX, y responde a la diversidad de formas estructurales, cada una con su especificidad misional propia, que se ha ido constituyendo a lo largo de las décadas, todas bajo la misma nomenclatura de «universidad». Así, el abanico de instituciones que se comprenden bajo el rótulo de «universidad» incluye, en el caso británico, entidades con ethos tan diversos como las que se engloban bajo el modelo Oxbridge, y su idea de la universidad como esencial para la preservación de la cultura de las minorías frente a la civilización de las masas. También incluye modelos como la Universidad de Londres, cuyo ideal cultural permitió la coexistencia de las ciencias puras y aplicadas, la economía política, las ciencias sociales y las humanidades. De igual modo, los colleges of advanced education, de carácter profesionalizante, que fueron elevados a estatus universitario en la década de los sesenta, también encarnan su idea de universidad, como también lo hacen las nuevas universidades creadas por la legislación de 1992, que unificó el sistema binario británico. A este variado mosaico de instituciones universitarias hay que añadir las entidades que desempeñan su labor mediante las nuevas tecnologías (ie. Open University, University of the Third Age, University of the Highlands and Islands). Por ultimo, también bajo el término de «Universidad» desarrolla su cometido la nueva University for Industry, cuyo ethos en gran medida, y a imitación de algunas instituciones norteamericanas similares, es proporcionar formación profesional específica para industrias concretas (Filmer, 1997, pp. 48-51). 
Podemos afirmar que los académicos modernistas admiten la diversidad institucional existente de modelos de universidad y la existencia de ideas diversas asociadas a dichos modelos. No obstante, ellos apuestan claramente por la categoría «más pura» de universidad, es decir, por aquella vinculada con la investigación básica y aplicada, y con la enseñanza de postgraduados (Filmer, 1997, pp. 52-53). Para los académicos modernistas, tanto en tiempos del Cardenal Newman como en la actualidad, la universidad es el «lugar de enseñanza del conocimiento universal» (Ker, 1999, p. 13). El constructo de «conocimiento universal» se equipara, en la lógica modernista, al «conocimiento liberal o filosófico» y, en suma, a la educación liberal. Por educación liberal los modernistas interpretan el cultivo real del intelecto, el conocimiento cuando está impregnado de razón, cuando está imbuido con «la idea del método, orden, principio y sistema para tener el hábito del método, de comenzar por una idea, seguirla, y distinguir lo que uno sabe de lo que no sabe» (Ker, 1999, p. 21). Dicha educación comprende, en suma, la adquisición de la facultad del juicio, la habilidad de distinguir prioridades y el poder de evaluar y realizar juicios normativos (Ker 1999, p. 24). La «cultura intelectual» es aprender a pensar y llegar a la excelencia intelectual. Esta cultura o filosofía presupone el conocimiento y la realización de amplias lecturas necesaria para adquirirlo, también la imaginación y la memoria, pero va mucho más lejos.

Frente a esta postura unánime de los académicos modernistas respecto a la naturaleza y cometidos de la universidad, destaca aquella otra, más indefinida y, desde nuestro punto de vista, más vulnerable, de los académicos partidarios de la universidad postmoderna. Podemos interpretar que, tal es la fragilidad de la idea de universidad de estos académicos que, en más de una ocasión, ellos se han planteado interrogantes drásticos relativos a «¿̇la muerte de la universidad?» (Smith y Webster, 1997, p. 106). Podemos decir que para los modernistas las transformaciones acaecidas en la universidad en el siglo XXI, analizadas en líneas anteriores, no modifican necesaria ni sustancialmente, el ethos ni la misión de la universidad. No obstante, para los académicos postmodernistas dichas modificaciones han alterado radicalmente la idea y la función tradicional de la universidad con las que, por otra parte, claramente ellos no comulgaban. Desde un prisma epistemológico, la visión postmodernista aduce que se ha producido una «devaluación de la cultura científica tradicional no solo en términos de decadencia de los cánones culturales e intelectuales que reflejaban los intereses y aspiraciones de los estudiantes de elite, sino también debido al creciente escepticismo respecto de las presunciones de universalismo que denotaban los valores de la racionalidad cognitiva» (Scott, 1997, p. 41). Académicos como Scott aluden a la deconstrucción de la cultura científica tradicional (tanto en el ámbito de sus valores cognitivos, prácticas sociales y formas institucionales) y de la emergencia de nuevos tipos de instituciones de conocimiento con patrones de socialización académica y profesional distintos a los típicamente universitarios y constituidos en modelos rivales de la universidad (Scott, 1997, p. 41). Al parecer de postmodernos como Scott y Bauman, el concepto de «conocimiento» se ha convertido en una categoría más amplia, que supera las definiciones y los ámbitos académicos y científicos y, por ello, a su entender, aparentemente ya no resulta claro que las universidades, tal y como están hoy constituidas, sean las instituciones más capaces de generar y manipular estas nuevas formas de conocimiento. 


\subsection{El debate sobre la teleología de la universidad europea}

La agenda postmoderna en la universidad está teniendo notables implicaciones en la teleología que impulsa esta institución en el siglo XXI. Antaño, en el marco de la tradición liberal de la universidad, esta institución poseía la finalidad básica del cultivo de la mente; de conseguir la cultura intelectual consistente en aprender a pensar y lograr la excelencia intelectual (Ker, 1999, p. 24). Desde los nuevos presupuestos postmodernistas la misión de la universidad ya no se entiende desde un prisma holista y desinteresado dirigido a la «educación de las mentes de los alumnos» (Ker, 1999, p. 25) sino que, vinculada ahora a una nueva cultura profesional, esta misión se concibe como una enseñanza preparatoria para dar respuesta a las demandas laborales y económicas, y a una nueva cultura laboral crecientemente diversificada.

El cambio de finalidad y de las motivaciones que impulsan el quehacer universitario es claramente visible en las aserciones que, en décadas diversas, han realizado a este respecto influyentes informes sobre la enseñanza superior británica. Tomamos el ejemplo británico como paradigma de la enseñanza superior europea porque, como acertadamente han expuesto diversos autores, «es más probable que las universidades europeas se orienten en la dirección de las británicas que al revés» (Smith y Langslow, 1999, p. 8). En este sentido, resulta de gran interés analizar las preocupaciones que se han cernido sobre la universidad británica en décadas diversas, a través de informes emblemáticos como, muy especialmente, el Informe Robbins (1963) y el Informe Dearing (1997).

Como han afirmado algunos autores, en los últimos cientos de años en Europa, las universidades tuvieron a la enseñanza como su cometido esencial (Watson, 2007, p. 82). La revolución que se operó con Humboldt reportó una segunda misión a la institución universitaria: la investigación, la producción de conocimiento. En el reciente cambio de milenio hemos atestiguado una «segunda revolución académica» (Watson, 2007, p. 82) que ha añadido una tercera misión a la enseñanza superior. Esta nueva misión consiste en hacer disponible y difundir el conocimiento innovador de la enseñanza superior, para el beneficio de la economía y de la entera sociedad. Para el Informe Dearing británico, esta tercera misión era la primordial: el Informe Dearing «está implicado con las cuestiones materiales y con el rol central de la enseñanza superior en la economía» (Niche, 1997. Citado por Watson, 2007, p. 94), sancionando una clara visión economicista de la enseñanza universitaria. Este tinte economicista de la función de la institución universitaria está también presente en el actual Anteproyecto de Ley Orgánica del Sistema Universitario (LOSU) de 2021 del Ministerio de Universidades español, al aludir a los «doctorados industriales», las "pasarelas con estudios de Formación Profesional superior», y la «ayuda de las universidades a nuestra sociedad y economía» (Ministerio de Universidades, 2021).

\subsection{El debate sobre las funciones sociales de la universidad europea}

La institución universitaria desempeña una serie de funciones sociales que son concebidas de manera diversa por los académicos modernistas y por los postmodernos. Así, podemos decir que los académicos modernistas entienden las funciones sociales de la universidad como sigue:

-Función de reproducción cultural -la universidad debe garantizar la continuidad de la tradición cultural, y constituir un medio en el que la cultura epistemológica pueda ser debatida, revisada y renovada mediante la innovación. 
-Función de docencia - la universidad tiene la responsabilidad social y cultural de enseñar a pensar críticamente respecto de la política y la sociedad (Filmer, 1997, p. 58). Junto a este cometido de formación de un pensamiento social crítico, esta postura es, con frecuencia, partidaria de asociar la excelencia epistemológica a la selección en el ingreso universitario, con un criterio basado en el método demostrado por los logros de los alumnos (Filmer, 1997, p. 58).

-Función de investigación - la concepción modernista se revela partidaria del desarrollo de una investigación pura y «desinteresada», atenta, de forma prioritaria, a los intereses epistemológicos del investigador.

Como valoración esencial del contenido de las funciones sociales de la universidad según son concebidas por los académicos modernistas cabe destacar que la independencia epistemológica que reclaman las funciones de docencia e investigación de este grupo de académicos, presupone una relación de completa autonomía de la universidad respecto de la sociedad. Se trata de una relación vertical de hegemonía de la primera respecto de la segunda, en tanto que es la universidad la que tiene la responsabilidad de dictar a la sociedad y a la política las prioridades de acción y el mejor camino de avance social hacia el futuro. Los académicos modernistas son conscientes de que hay unos rasgos en la sociedad actual que parecen reclamar una redefinición de estas funciones. No obstante, esta postura académica se muestra firme en la resolución de que las instituciones de enseñanza superior deben resistirse a los «roles reduccionistas» a que la política y la economía parecen abocarla, y afirman que la universidad debe «mantener una visión clara de su lugar y su rol en la sociedad contemporánea -visión que depende de la habilidad de estas instituciones en desarrollar una provisión desinteresada de educación, formación e investigación» (Filmer, 1997, p. 57). Como puede apreciarse, la postura modernista, lejos de representar una visión caduca y desfasada de la universidad, constituye una apuesta clara y decidida por un rol atemporal de la universidad como institución que lidera la sociedad. Por otra parte, esta visión también abraza nuevas funciones sociales de la universidad contemporánea que no entran en conflicto con el rol de liderazgo de la universidad, como son la función de educación permanente de la universidad, la función de educación para el ocio, así como la función universitaria de internacionalización y creación de redes europeas de excelencia.

Los académicos postmodernos poseen una concepción de las funciones sociales de la universidad bien diversa respecto de los autores anteriores. Este grupo de estudiosos sostiene que, a comienzos del siglo XXI, nos hallamos en una situación de ruptura total con la era de la modernidad tardía. Desde su perspectiva, esta escisión ha ocasionado cambios sustanciales en la institución universitaria. En concreto, entre las modificaciones que autores como Scott señalan que se han operado en la universidad como consecuencia de la era postmoderna destacan el nuevo carácter funcionalista de las categorías definitorias de la enseñanza superior frente al típicamente esencialista de antaño -ahora considerado anacrónico-; la sustitución de las tradiciones de relación íntima en la enseñanza superior británica por sistemas burocráticos de orientación y garantía de la calidad; la reducción del perfil y las prácticas colegiadas y del ideal de la comunidad universitaria y la potenciación, en su lugar, del poder y el perfil de los gestores institucionales; la erosión de las demarcaciones entre la enseñanza superior y otras formas de educación; la competición del criterio tradicional de acceso a la enseñanza superior mediante la selección académica con el de la elección del consumidor de la institución superior más 
atractiva y, en suma, el énfasis en el rendimiento y la consecución de objetivos (Scott, 1997, pp. 38-41). Así, pues, con vistas a dar respuesta a la nueva configuración de la sociedad, la institución universitaria debe adoptar, según estos autores, unas nuevas funciones sociales distintas de las tradicionalmente desempeñadas:

-Función de producción de jerarquías sociales (ya no de reproducción).

-Función de enseñanza e investigación: el desarrollo de estas funciones no se entiende ahora desde un prisma holista y desinteresado dirigido a la «educación de las mentes de los alumnos» (Ker, 1999, p. 25), sino íntimamente vinculado y en respuesta a las demandas laborales y a una nueva cultura laboral de ocupaciones más diversificadas y difusas que las racionalistas y burocratizadas de antaño y que demandan un currículo orientado a la potenciación de destrezas, aptitudes y actitudes.

-Función de educación permanente (Scott, 1997, p. 45; Fernández, 1999, p. 221; Gellert, 1993, p. 240): esta función es destacada y promovida por todos los estudiosos. No obstante, los académicos modernistas la entienden, primordialmente, como la oportunidad que la institución universitaria brinda a toda la sociedad para participar en la sociedad del conocimiento. Y, por su parte, para postmodernos como Scott esta función supone «no solo proporcionar a los futuros graduados las destrezas del aprendizaje a lo largo de la vida -curiosidad, flexibilidad y adaptabilidad- sino potenciar el desarrollo de una nueva mentalidad irónica, intuitiva e instantánea" (Scott, 1997, p. 45).

-Función de educación para el ocio: esta función es adoptada, sin ambages, por ambas posturas académicas. Los diversos académicos entienden que la educación universitaria está «crecientemente incorporada en el sector más amplio de «aprendizaje y ocio», el cual constituye el $13 \%$ del producto nacional bruto en Inglaterra» (Scott, 1997, p. 46). La enseñanza superior, pues, ya no ejerce solo una función meramente preparatoria y preliminar para el mundo laboral, sino que muchas personas acuden ahora a ella como antídoto al trabajo, por lo que la universidad debe atender al desarrollo de destrezas personales, sociales y vitales en las personas que acuden a la misma (Scott, 1997, p. 47).

Hay que destacar, como resumen de la visión postmoderna de las funciones sociales de la universidad, que sus concepciones proponen y suponen una relación de la universidad con la sociedad diametralmente opuesta a aquella defendida por la visión anterior. Así, la postura postmoderna sugiere una relación básicamente horizontal de la universidad con la sociedad, en la que la primera está supeditada a la agenda política de la sociedad, y a los imperativos económicos neoliberales actuales. Con esta propuesta, desde mi punto de vista, la universidad se ve desprovista de su ethos más característico y propio de liderazgo y conducción de la sociedad. Desde esta visión se entiende la pregunta que, con frecuencia, se plantean los postmodernos: ¿la muerte de la universidad?, dado que, si la universidad es desprovista de su ethos específico, esta institución se asemeja a cualquier otra institución en la realización de una función de enseñanza y profesionalización atendiendo a los criterios del mercado. 


\subsection{El debate sobre la epistemología de la universidad europea}

El sometimiento de las economías nacionales a los procesos de la globalización, ha generado una creciente presión por parte de la industria y el comercio en los gobiernos para que estos proporcionen incentivos a las universidades dirigidos a la obtención, por parte de estas, de «productos» e innovaciones comercializables (cf. King, 2004, p. 54). Las políticas nacionales de educación superior parecen estar garantizando que la financiación pública disponible para actividades discrecionales o marginales sea dirigida a ámbitos de investigación, bien focalizados directamente en innovación de productos, bien vinculada con áreas de innovación en grandes empresas globales, como las de alta tecnología (King, 2004, p. 55). En todos los ámbitos de la política universitaria, y de la misma institución universitaria, parece estarse imponiendo el paradigma de la universidad empresarial (King, 2004, p. 56), regido por lo que algunos autores han denominado el «capitalismo académico» (Slaughter y Leslie, 1997, citado por King, 2004, p. 54). Por «capitalismo académico» se entiende el «incremento de las relaciones de mercado en la producción y distribución de conocimiento académico» (Slaughter y Leslie, 1997, citado por King, 2004, p. 54). Hay autores que se refieren a esta nueva dinámica de los procesos universitarios como una sometida a los imperativos de rendimiento, "performativity», impuestos por instancias gubernamentales, a partir de los cuales la tradicional autonomía universitaria ha experimentado una disminución o «atenuación en sus relaciones económicas, pedagógicas, personales y de investigación» (Cowen, 1996, p. 245).

Como afirma el académico Cowen, el análisis teórico de las razones de la emergencia del paradigma postmodernista no ha sido nunca, realmente, del interés ni de las organizaciones internacionales ni de los gobiernos, que son los que, en definitiva, instan, ambos, a la reforma de los sistemas universitarios de modo que la competitividad internacional basada en el conocimiento y las aplicaciones de este en la economía produzcan un exitoso rendimiento. Desde el momento en que se ha operado la centralidad del conocimiento como bastión de la nueva economía internacional de la información y, más visiblemente, desde las décadas de los ochenta y noventa, se ha operado un movimiento de reforma universitaria, más manifiesto en países como los EE.UU., el Reino Unido, Canadá y Australia, dirigido a rediseñar los sistemas universitarios según criterios de eficiencia y relevancia. El concepto de eficiencia «incluye la medición de la producción universitaria de conocimiento, y el test de relevancia implica la valoración de la utilidad del contenido de la docencia y la investigación para la economía nacional» (Cowen, 1996, p. 246). Desde estos parámetros, organismos como la OCDE han apostado por promocionar, de preferencia, ciertos ámbitos científicos frente a otros (ie. la genética, la electrónica, la física, la bioquímica). Ya Lyotard, a comienzos de la década de los ochenta, estableció una cierta jerarquización entre disciplinas, estableciendo la «mayor importancia de unas ciencias frente a otras» (ibidem, p. 248). Así, Lyotard expuso que «(...) en los últimos cuarenta años las ciencias y tecnologías que han ostentado el liderazgo son las relacionadas con el lenguaje: problemas de comunicación y cibernética, teorías modernas de álgebra e informática, computadoras y sus lenguajes, problemas de almacenamiento de la información y bases de datos, telemática y la perfección de terminales inteligentes» (Lyotard, 1984, pp. 3-4).

De igual modo, en términos epistemológicos, y en lo que atañe a la producción del conocimiento, autores como Gibbons (1994) afirman que se está produciendo un tránsito del «conocimiento Tipo 1» al «conocimiento Tipo 2». El «conocimiento Tipo 1» implica 
un conocimiento generado en un contexto disciplinar y cognitivo. Se trata de un conocimiento generado dentro de una estructura vertical. Frente a esa forma de hacer conocimiento, el «conocimiento Tipo 2» resulta de la multiplicación del conocimiento en la sociedad contemporánea y el crecimiento heterogéneo en el número y tipos de ámbitos en los que el conocimiento se produce y se utiliza. El «conocimiento Tipo 2» es uno de rasgos transdisciplinares y heterogéneos, e implica una estructura horizontal del conocimiento. Según algunos autores, este tipo de conocimiento tiene los rasgos de «no jerárquico, plural, transdisciplinar, cambiante y socialmente adaptado a las necesidades diversas de los estudiantes y de las prioridades de la industria» (Smith y Webster, 1997, p. 104).

Los rasgos epistemológicos de la universidad postmoderna tienen, como no podía ser de otra manera, hondas repercusiones en el ámbito de la investigación universitaria. En primer lugar, cabe decir que se aprecia una creciente convergencia entre la investigación básica y aplicada (King, 2004, p. 53). En términos generales, la tendencia apunta a una menor financiación de la docencia universitaria y a un incremento de aquella destinada a la investigación aplicada. La OCDE, en este sentido, ha instado a la acotación, por motivos económicos, de la investigación básica, y a la potenciación preferente de la investigación aplicada (idem). De igual modo, se potencian crecientemente las actividades que fomentan el potencial universitario de obtener financiación externa (King 2004, p. 54). En segundo lugar, cabe decir que la globalización ha potenciado la comercialización de elementos clave de la investigación universitaria (idem). Se han incrementado enormemente las relaciones de mercado en la producción y distribución del conocimiento académico, proceso especialmente manifiesto en las disciplinas vinculadas con las ciencias tecnológicas. Esto es ilustrado por la existencia, desde la década de los ochenta, de centros y departamentos interdisciplinares (ciencia óptica, ciencia cognitiva, etc.) caracterizados por sus actividades comerciales y que reflejan un intercambio emergente entre la empresa y la necesidad de las universidades de compensar el declive de la financiación pública mediante la búsqueda de mayores oportunidades de mercado (King, 2004, p. 54). En tercer lugar, puede decirse que, con relación a la investigación universitaria, la globalización ha potenciado una nueva función de la universidad, que radica en la internacionalización y la creación de redes europeas de excelencia y de desarrollo de una colaboración interestatal en la investigación, así como el análisis en la investigación de temáticas de interés mundial como el medioambiente, la inmigración o los derechos humanos.

Lo que parece claro es que, actualmente, en la sociedad occidental hay una compleja coexistencia de ambas tendencias. Por un lado, es clara la orientación empresarial que se quiere dotar a la universidad, a través del establecimiento de vínculos con el ámbito empresarial e industrial. Por otro lado, es manifiesta la reivindicación de muchos sectores relativa al hecho de que la actual sociedad del conocimiento precisa, más que nunca, de la categoría laboral de los denominados «analistas simbólicos», personas cuya excelencia analítica de la información y del conocimiento es solo aportada a través de una sólida y prolongada formación académica. Podemos interpretar que la primera tendencia es la que prima en la retórica política de las entidades y organismos internacionales actuales (Unión Europea, OCDE), mientras que la segunda es instada, sobre todo, por las entidades universitarias particulares y algunos ámbitos definidos y acotados del mundo laboral. 


\section{El impacto de Bolonia en la universidad eu- ropea y su incidencia en el debate modernis- mo-postmodernismo}

Uno de los efectos más notables de la globalización en el ámbito internacional se ha cifrado en el establecimiento de un marco de organizaciones internacionales, a través de las cuales se ha configurado un «gobierno sin Gobierno» (Dale, 2007, 51). Sobresalientes entre esas instituciones internacionales se han revelado el FMI, la OCDE, el G-7, el Banco Mundial y la UE. En este epígrafe nos vamos a referir a dos de ellas, de particular relevancia en el tema que nos ocupa: la OCDE y la UE.

Nuestra referencia, breve, a la OCDE en este análisis se justifica en tanto que esta institución está revelando un impacto sin precedentes en los niveles educativos que preceden a la enseñanza superior en los sistemas educativos que pertenecen a la misma. Así, numerosos académicos coinciden en que, en el actual momento de globalización «la OCDE ha devenido crecientemente un actor político por derecho propio» (Henry et all, 2001) en política nacional e internacional. Desde la lógica neoliberal que preside el discurso educativo de la OCDE, esta institución analiza las cuestiones educativas desde un «fundamentalismo económico» (Rizvi and Lingard, 2009) y desde una dimensión esencialmente técnica, vinculada a estadísticas e indicadores internacionalmente comparables, respecto de los cuales la OCDE ha adquirido gran maestría. El programa PISA de la OCDE ha obtenido un impacto sin precedentes en política educativa internacional, y es una muestra del enfoque numérico y económico de las actuales propuestas educativas de los organismos internacionales. Los resultados PISA son tomados muy en serio por las naciones participantes, como medida de la eficacia de sus sistemas educativos y la calidad de su capital humano. Lo que en este análisis nos interesa de la OCDE y de PISA es la visión educativa propuesta y difundida por sus promotores. Así, Andreas Schleicher, Director del Departamento de Análisis e Indicadores de la OCDE, en el prólogo que ha escrito al conocido Informe McKinsey establece que «el mundo es indiferente a la tradición» y que «el éxito llegará a aquellos individuos y países dispuestos a adaptarse y abiertos al cambio» (Schleicher, 2007, 6). Estas palabras de Schleicher permiten apreciar y concluir el hecho de que el máximo promotor del Programa PISA es, sobre todo, un técnico experto en estadísticas e indicadores internacionalmente comparables, pero no lo es tanto en el conocimiento de los elementos pedagógicos que integran la cultura escolar de los diversos países que han participado en las pruebas PISA. Como es sabido, hay dos países que se sitúan en los primeros puestos del ranking establecido por el Programa PISA, Finlandia y Corea del Sur, que poseen una cultura escolar de reconocido tradicionalismo, que han demostrado que muchos de los elementos de la tradición educativa funcionan en el siglo XXI.

La Unión Europea (UE) es otra de las instituciones supranacionales de gran incidencia en los primeros años del siglo XXI. Particularmente relevante a nivel mundial en los tiempos actuales es su impulso del Espacio Europeo de Enseñanza Superior. En este proceso de armonización de sistemas de enseñanza superior nos hallamos profundamente involucrados e interesados no solo los países de la UE, sino todos aquellos demás continentes interesados en diseñar un sistema de enseñanza superior compatible con el europeo. Según el Informe Trends V de la European Universities Association (EUA), «el legado más significativo de este Proceso será un cambio de paradigma educativo por todo 
el continente» (Crosier, Purser y Smidt, 2007, 7). Como ocurre con los dirigentes educativos de la OCDE, también los asesores educativos del Proceso de Bolonia revelan poseer la extendida atribución de equivalencia, no enteramente empírica, entre excelencia e innovación y cambio. No es infrecuente asistir a explicaciones, por ejemplo, del éxito educativo de Corea del Sur de autores como Watkings y Biggs (1996), los cuales hablan de «la paradoja del estudiante asiático» para referirse a la aparente contradicción ante el empleo en este país de elementos pertenecientes al paradigma de la educación formal, y el éxito académico de los estudiantes del este asiático en pruebas internacionales de educación como el TIMSS del IEA y el PISA de la OCDE. Académicos como Crook han evidenciado la falta de pruebas empíricas que demuestren la mayor o menor bondad o eficacia del paradigma de la pedagogía formal sobre el de la pedagogía progresista, o de este respecto al primero (Crook, 2011).

Sin lugar a dudas, el inicio y desarrollo del Proceso de Bolonia ha reavivado el debate sobre el ethos moderno o postmoderno de la institución universitaria europea. Se trata de un debate apasionante sobre el que han aparecido obras bibliográficas de gran interés, como la editada por Anthony Smith y Frank Webster en el año 1997.

Desde un punto de vista teórico y conceptual podemos afirmar que los teóricos del Proceso de Bolonia se sitúan en una postura intermedia entre las perspectivas modernas y postmodernas de la concepción de la universidad. Así, la Declaración de la Sorbona subraya el «todavía papel imprescindible que desempeñan las universidades» en la creación de la Europa de los conocimientos (Declaración de la Sorbona, 1998). Este documento, y aquellos que le siguen doctrinalmente en la creación del EEES, como la Declaración de Bolonia de 1999, afirma el «papel protagonista de las instituciones de enseñanza superior europeas en la construcción del EEES» (Declaración de Bolonia, 1999). Desde un punto de vista teórico, la relevancia que estos documentos conceden a la universidad aleja a los teóricos del Proceso de Bolonia de posturas empíricamente postmodernas, las cuales hablan de la «desinstitucionalización del conocimiento» (Scott, $1997,36)$ y, a la postre, finalizan sus reflexiones con interrogantes como «¿̇la muerte de la universidad?» (Smith y Webster, 1997, 106).

Otro de los parámetros de los planteamientos del Proceso de Bolonia en el que podemos percibir claramente la naturaleza moderada (a priori) de esta reforma, radica en su reconocimiento del valor y del legado de la tradición cultural europea. Así, documentos como el Comunicado de Berlín de 2003 establecen un claro equilibrio entre tradición y reforma al afirmar que «el objetivo (de la emergente Área de Educación Superior Europea) es preservar tanto la riqueza cultural europea como su diversidad lingüística, basada en la herencia de muy diversas tradiciones, y fomentar su potencial de innovación» (Comunicado de Berlín, 2003). No cabe duda que este planteamiento históricamente continuista del Proceso de Bolonia, no solo aleja el discurso de Bolonia de algunas propuestas postmodernas esencialmente rupturitas, sino que diseñan una ruta educativa de futuro altamente recomendada por académicos finlandeses que aconsejan, par el siglo XXI, un delicado equilibrio entre tradición y reforma, y la introducción de solo aquellos elementos educativos de validez probada y siempre que no modifiquen sustancialmente nada que se esté revelando eficaz en el sistema (Simola, 2005; Räsänen, 2006).

Por las cuestiones a las que hemos aludido, no podemos sino calificar la naturaleza y la concepción teórica del Proceso de Bolonia como de moderada y de educativamente acertada, pues creemos que solo se puede construir un futuro sólido en educación, historia y cultura, si está conveniente y firmemente vertebrado en la tradición que lo sustenta. 
En lo que atañe a la teleología del Proceso de Bolonia, las cosas no están tan claras. Hay una serie de objetivos de índole general, que suscitan el consenso general de la comunidad universitaria europea. Se trata de los objetivos de que el EEES potencie «los valores democráticos, la diversidad de culturas y lenguas, y la diversidad de los sistemas de educación superior» (Declaración de Praga, 2001). Otros objetivos del EEES, explícitos en diversos documentos, también suscitan el consenso general, dado que ello contribuye a potenciar la democratización de la educación y la internacionalización de la misma. Son los objetivos de la movilidad, la cooperación universitaria, la garantía de la calidad, la promoción de la cohesión social y la reducción de las desigualdades sociales y de género, el vínculo del área de la educación superior europea y el área de investigación europea, y la potenciación del Aprendizaje a lo Largo de la Vida.

Hay otra serie de objetivos, fundamentalmente de índole estructural, que implican cambios sustanciales de los sistemas nacionales de educación superior, pero que han sido, a la postre, aceptados por la comunidad universitaria dado que contribuyen al fin de la creación de un espacio común de enseñanza superior europea que puede potenciar internacionalmente nuestros sistemas. Son los objetivos de adopción de un sistema basado en tres ciclos, la puesta a punto de un sistema de créditos ECTS, y demás elementos dirigidos a garantizar la compatibilidad y la comparabilidad de los sistemas nacionales de educación superior.

Por último, hay una serie de objetivos metodológicos y curriculares que afectan de lleno la entidad de los procesos educativos, que han tratado de ser impuestos a partir del Comunicado de Londres del año 2007 (en los documentos anteriores del Proceso de Bolonia no aparecían) y sobre los cuales hay una notable incertidumbre por parte de un sector de la comunidad universitaria europea. Frente al carácter esencialmente estructural de las medidas contenidas en los documentos marco anteriores (Declaraciones de la Sorbona, 1998; de Bolonia, 1999; de Praga, 2001; de Berlín, 2003, y de Bergen, 2005), el Comunicado de Londres de 2007 insiste (y así se especifica también en todos los documentos posteriores a este) en «la transición hacia una educación superior centrada en los estudiantes y no en una educación centrada en el profesor». En esta misma línea, los Comunicados de Lovaina (2009) y de Budapest (2010) insisten en la necesidad de una reforma curricular centrada en las competencias profesionales y en el desarrollo de resultados de aprendizaje. Tal es la insistencia actual de los últimos documentos del Proceso de Bolonia en una metodología y un currículo competencial y profesionalizante, que los informes de la European Universities Association, EUA, que informan las reuniones de Ministros de Educación europeos (EUA Trends Report, del Trends I, junio 1999, al Trends VI, 2010) hablan de que «el legado más significativo del proceso será un cambio de paradigma educativo por todo el continente» (Crosier, Purser, Smidt, 2007, 7), y de una «metamorfosis» de la educación superior europea (Sursock \& Smidt, 2010, 15).

\section{Conclusiones}

Podemos afirmar que, desde hace unos años, los debates académicos en Educación Comparada han adoptado un creciente tono postmoderno. En lo que atañe a la educación globalmente considerada, la ascendencia del postmodernismo revela una cierta «fragmentación»e «incoherencia» que revela ciertas «inestabilidades y contradicciones». La aún vulnerabilidad e inestabilidad doctrinal de las propuestas educativas postmodernas promueven y permiten la coexistencia de esta polaridad en propuestas educativas. Hoy 
más que nunca se revelan ciertas las palabras del académico Holmes en relación con la coexistencia de paradigmas educativos en el ámbito de las ciencias sociales en que se inscribe la ciencia de la Educación Comparada.

En lo relativo a la institución universitaria, empieza a despuntar la postura más indefinida y vulnerable de los académicos partidarios de la universidad postmoderna (Scott, 1997; Bauman, 1997) que incluso se han planteado interrogantes drásticos relativos a «¿la muerte de la universidad?» (Smith y Webster, 1997). En este sentido, frente a las funciones tradicionales de la universidad, que se cifraban en una función de reproducción cultural, de investigación pura y desinteresada, y de docencia basada en la enseñanza a pensar críticamente respecto de la política y la sociedad (Filmer, 1997, pp. 52-53), la universidad postmoderna se vertebra en funciones de producción, de enseñanza e investigación en respuesta a las demandas laborales, de educación permanente y de educación para el ocio (Scott, 1997, p. 45).

Desde un prisma epistemológico la nueva universidad construye su saber desde el reconocimiento del Modo 2 de creación del conocimiento, el cual se establece en un contexto de aplicación, heterogeneidad, ausencia de jerarquía, transitoriedad y responsabilidad social. Un aspecto nuclear que necesita revisión académica y política, máxime cuando constituye el punto crucial de desacuerdo entre los académicos modernistas y postmodernos, es el relativo a las consecuencias de dotar a la universidad de funciones como la de innovación y la exigencia que esta función con lleva de cooperación estrecha con la industria. Quizá esa función es más propia para ser desarrollada por otras instituciones, no universitarias, de enseñanza superior, que deben ser promovidas numérica y económicamente. Una de las graves consecuencias de potenciar la cultura profesional en la universidad frente a la académica radica en la relegación a un segundo plano de las humanidades y las ciencias sociales, y en la obligatoriedad de desarrollar de preferencia la vertiente más profesional de estas disciplinas. El perjuicio de ambas medidas a nivel social es incalculable: a las humanidades y las ciencias sociales compete, más que a otras disciplinas, el diseño del modelo de sociedad y de hombre para el siglo que acaba de comenzar, y más en estos tiempos relativistas y postmodernos en que nos encontramos. Sin esa orientación, fundamental en los ámbitos político y social, la misma sociedad queda al dictado de la economía como única vigía social, con todos los riesgos, limitaciones y empobrecimiento social y moral que esto supone. La respuesta de la universidad a la globalización no puede suponer, en ningún caso, la abdicación, por parte de esta institución, de su ethos constituyente y siempre vigente: la universidad es la entidad rectora, por excelencia, de los destinos de la sociedad en la que se inserta.

En otro orden de cosas, destacar que, pese a la aparente coherencia de las medias políticas propuestas por la Unión Europea en materia universitaria, el análisis de los procesos actualmente operantes en el entorno universitario, y los que se adivinan para un futuro próximo, detecta ciertas fisuras y contradicciones en materias de enorme relevancia social como, muy particularmente, el potencial democratizador o selectivo de las futuras instituciones universitarias, dadas las propuestas a favor de la privatización de la universidad que parecen derivarse de la globalización. Una mirada a la enseñanza superior norteamericana, modelo al que más certeramente nos encaminamos, puede aportar claves importantes en la resolución europea de estas dicotomías.

Quizá no exista aún una teoría postmoderna de la educación explícitamente articulada como tal, en la línea que afirma el académico Green (1994). Pero, desde mi particular opinión académica, parece claro que estamos asistiendo a un cambio de ciclo histórico 
en el ámbito educativo internacional y global que se orienta al profesionalismo y a la potenciación de destrezas y competencias en el currículo académico de todos los niveles educativos de los sistemas, frente al prolongado período anterior que ha potenciado los contenidos de cuño teórico, académico, abstracto y doctrinal en los mismos. Si bien la literatura que analiza el impacto del postmodernismo en educación no ha llegado aún a vertebrar dicha teoría cuya existencia niega el académico Green, parece, en mi opinión y reflexión académicas, que con el postmodernismo se ha iniciado una era educativa que apuesta internacionalmente sin ambages por implantar las propuestas educativas de la Escuela Nueva en educación, muy particularmente sus propuestas paidocéntricas, de relaciones horizontales en la relación docente-discente, de predominio de la actividad, y de la evaluación continua en todo el sistema. Incluso países muy seguros de su tradición académica como Alemania y Finlandia, han sucumbido a este viraje educativo en sus Gymnasium. Muchas de las aportaciones educativas y formativas de la Escuela Nueva se revelan brillantes. No obstante, desde también la innegable bondad de muchos elementos educativos del paradigma de la pedagogía formal, creemos óptima la combinación de elementos de ambos paradigmas (formal y progresista), que equilibre y establezca procesos educativos sin mermas ni polarizaciones sesgadas.

Hay otro aspecto que deseamos comentar en estas conclusiones. Y es la dispar dirección operada, de arriba abajo con el Proceso de Bolonia de la UE, y de abajo arriba con el estudio PISA de la OCDE, de impacto educativo en los sistemas mundiales de educación. El Proceso de Bolonia de la UE ha seguido la lógica histórica de reforma de la educación desde la universidad. En la concepción del Proceso de Bolonia, que es el pensamiento imperante históricamente, la universidad es la institución mentora de los restantes niveles educativos, e influye en el carácter de los mismos en términos de contenidos y de metodologías educativas. De igual modo, en este proceder, es la universidad (muy particularmente a través de las ciencias humanas y sociales) la que concibe y diseña el modelo futuro de hombre y de sociedad. Este proceso experimenta una drástica ruptura y cambio con la evaluación PISA de la OCDE. En dicho proceso de la OCDE, se opera el estudio competencial de los niveles obligatorios de los sistemas educativos, los cuales tienen la suficiente entidad y relevancia por sí mismos para emitir una valoración de la idoneidad educativa de estos niveles educativos, más allá y de forma independiente de lo que puede reclamar la institución universitaria. Pero ni dichos niveles educativos de forma aislada, ni la institución universitaria sometido al dictado de la economía, pueden ya concebir el modelo futuro deseable de la sociedad humana.

En este proceso cultural y educativo hay elementos positivos y otros que se ven desmejorados. Entre los aspectos favorables y que salen reforzados de este proceso se halla el sector de la formación profesional de los diversos países, particularmente España, que desde hacía ya demasiado tiempo precisaba una gran reforma de esa sección del sistema educativo. Entre los aspectos desfavorables se encuentra el hecho de la disminución del rol director y rector de la universidad (y de las ciencias humanas y sociales) en el diseño de los destinos sociales. La institución universitaria está actualmente experimentando un proceso contradictorio consistente en, de forma simultánea, el alejamiento y la reducción de sus tareas formativas. Así, por un lado los estudiantes casi de forma automática proceden a cursar el nivel de máster tras el estudio de sus reducidos grados. De igual modo, cada vez es mayor el número de egresados del nivel de máster que se encamina a estudios de doctorado. Por el contrario, las instituciones universitarias vienen de forma creciente diseñando y ofertando la estructura institucional de los microgrados, como 
formación universitaria rápida y profesionalizante, que busca finalizar de forma breve y concisa el proceso educativo de este nivel de la enseñanza.

Como reflexión esencial frente a estos nuevos planteamientos postmodernos en educación podemos colegir que la teoría de la postmodernidad posee aún muchas vulnerabilidades y carencias. Ello hace especialmente recomendable la propuesta de una relación equilibrada y continuista, no rupturista, con la etapa de la Modernidad. Coincidimos con Habermas (1988) en su reflexión de que el proyecto de la Modernidad está aún inacabado, y de que la crítica postmoderna supone un correctivo al proyecto de la Modernidad que puede ser perfectamente incorporado a dicho proyecto, modificándolo, pero no invalidándolo ni sustituyéndolo. Con dichos correctivos, el proyecto de la Modernidad y muchas de sus metanarrativas siguen siendo enteramente válidas. El postmodernismo debería guardar un equilibrio con la Modernidad, pues es obvio que no todo el proyecto moderno es inválido.

\section{Referencias bibliográficas}

Anderson, P. (2000). Los orígenes de la postmodernidad. Madrid: Akal.

Ball, S. (1998). Big policies/small world: An introduction to international perspectives in education policy. Comparative Education, 34(2), 119-130.

Ball, S. J. (2006). Education Policy and Social Class. The selected works of Stephen J. Ball. London: Routledge.

Bauman, Z. (1997). Universities: Old, New and Different. En A. Smith \& F. Webster (Eds.), The Postmodern University? Contested visions of Higher Education in Society. Buckingham: SRHE and Open University Press.

Bertens, H. (1995). The idea of the postmodern: a history. London: Routledge.

Carter, J. (1998). Postmodernity and the Fragmentation of Welfare. London: Routledge.

Comisión Europea (1993). ¿Qué futuro para la educación superior en Europa? Reacciones al Memorándum. Bruselas: Comisión Europea.

Comisión Europea (2003). El papel de las universidades en la Europa del conocimiento. Bruselas: Comisión Europea.

Comisión Europea (2006). Delivering on the modernization agenda for universities: education, research and innovation. Bruselas: Comisión Europea.

Committee of Higher Education (1963). Higher Education. Report of the Committee appointed by the Prime Minister under the Chairmanship of Lord Robbins 19611963. London: HMSO.

Comunicado de Berlín (2003). Educación Superior Europea. Berlín: Conferencia Ministros Educación Superior.

Cowen, R. (1996). Performativity, Postmodernity and the University. Comparative Education, 32, 245-258. 
Cowen, R. (2003). Agendas of attention: A response to Ninnes and Burnett. Comparative Education, 39(3), 299-302.

Crook, D. (2011). Standards in English education: An enduring historical issue. Revista Española de Educación Comparada, 18.

Crosier, D., Purser, L. \& Smidt, H. (2007). Trends V: Universities shaping the European Higher Education Area. Brussels: EUA.

Dale, R. (2007).Specifiying Globalization Effects on National Policy. En B. Lingard \& J. Ozga (Eds.). Education Policy and Politics. London: Routledge.

Declaración de Bolonia (1999). El Espacio Europeo de la Enseñanza Superior. Bolonia: Conferencia Ministros Educación Superior.

Declaración de Praga (2001). Hacia el Área de la Educación Superior Europea. Praga, Conferencia Ministros Educación Superior.

Declaración de la Sorbona (1998). Declaración conjunta para la armonización del diseño del Sistema de Educación Superior Europeo. París: Ministros de Educación Superior.

Fernández, J. M. (1999). Manual de política y legislación educativas. Madrid: Editorial Síntesis.

Filmer, P. (1997). Disinterestedness and the Modern University. En A. Smith \& F. Webster (Eds.), The Postmodern University? Contested visions of Higher Education in Society. Buckingham: SRHE and Open University Press.

García Garrido, J. L. (1996). Fundamentos de Educación Comparada. Madrid: Dykinson.

García Garrido, J.L. (1998). Un nuevo horizonte para la educación secundaria. En AA.VV., Aprender para el futuro. La educación secundaria, pivote del sistema educativo. Madrid: Fundación Santillana.

García Ruiz, M. J. (2011). La Educación Comparada: una disciplina entre la Modernidad y el Postmodernismo. Revista Latinoamericana de la Educación, 2, 40-59.

Gellert, C. (Ed.) (1993). Higher Education in Europe. London: Jessica Kingsley Publishers.

Gibbons, M. (2004). Globalization, Innovation and Socially Robust Knowledge. En R. King (Ed.), The University in the Global Age. Basingstoke: Palgrave Macmillan.

Green, A. (1994). Postmodernism and state education. Journal of Education Policy, 9(1), 67-83

Habermas, J. (1988). Modernidad versus Postmodernidad. En J. Picó (Ed.), Modernidad y Postmodernidad. Madrid: Alianza Editorial.

Henry, M. et al. (2001). The OCDE, globalization and education policy. Oxford: Pergamon. 
Jarvis, P. (1996). Continuing education in a late-modern or global society: towards a theoretical framework for comparative analysis. Comparative Education, 32(2), 233-44.

Jones, B. (1995). Sleepers Wake! Technology and the Future of Work. Melbourne: Oxford University Press.

Ker, I. (1999). Newman's Idea of a University. A Guide for the Contemporary University?. En Smith, D. y Langslow, A. K. (Eds.), The Idea of a University. London, Jessica Kingsley Publishers.

King, R. (2004). The university in the Global Age. Basingstoke, Palgrave Macmillan.

Kogan, M. y Hanney, S. (2000). Reforming higher education. London, Jessica Kingsley Publishers.

Kumar, K. (1997). The Need for Place. En A. Smith \& F. Webster (Eds.), The Postmodern University? Contested visions of Higher Education in Society. Buckingham: SRHE and Open University Press.

Kunitz, S. J. (Ed.). (1955). Twentieth Century Authors - First Supplement. New York.

Lyotard, J. F. (1984). The Postmodern Condition. A Report on Knowledge. Manchester: University of Manchester Press.

Mehta, S. \& Ninnes, P. (2000). Postpositivist debates and comparative education: resistance, reinvention, revolution. Paper presented at the CIES annual conference. San Antonio, Texas. Comparative Education, 39(3), 279-297.

Ministerio de Universidades (2021). Anteproyecto de la Ley Orgánica del Sistema Universitario a los efectos previstos en el artículo 26.4 de la Ley 50/1997, de 27 de noviembre, del Gobierno. Madrid: Ministerio de Universidades.

National Committee of Inquiry into Higher Education (NCIHE). (1997). Higher Education in the Learning Society (The Dearing Report). London: HMSO.

Ninnes, P. \& Burnett, G. (2003). Comparative Education research: poststructuralist possibilities.

Ninnes, P. \& Mehta, S. (2000). Postpositivist theorizing and research: challenges and opportunities for Comparative Education. Comparative Education Review, 44(2), 205-212.

Olson, C. (1966). Projective Verse. En R. Creeley (Ed.), Selected Writings. New York.

Paulston, R. (1999). Mapping comparative education after postmodernity. Comparative Education Review, 43(4), 438-63.

Peters, M. (Ed.) (1998). Naming the multiple: poststructuralism and education. Connecticut: Bergin and Garvey.

Puelles Benítez, M. de (1993). Estado y educación en las sociedades europeas. Revista Iberoamericana de Educación, 1. 
Räsänen, R. (2006). Quality Education - A Small Nation's Investment for Future. Oulu: Faculty of Education. Teaching material for teacher education. Document not published.

Rizvi, F. and Lingard, B. (2009).The OCDE and Global Shift in Education Policy. En Cowen, R. \& Kazamias, A.M. (Eds). International Handbook of Comparative Education. London: Springer.

Rust, V. D. (1991). Post-modernism and its comparative implications. Comparative Education Review, 35(4)

Scott, P. (1997). 'The Postmodern University?'. En A. Smith \& F. Webster. (Eds.), The Postmodern University? Contested Visions of Higher Education in Society. Buckingham: SRHE and Open University Press.

Simola, H. (2005). The Finnish miracle of PISA: historical and sociological remarks on teaching and teacher education, Comparative Education, 41(4). Pp. 455-470.

Schleicher (2007). Introduction. In McKinsey Report. How the world's best-performing school systems come out on top. Mckinsey \& Company.

Smart, B. (1992). Modern Conditions, Postmodern Controversies. London: Routledge.

Smith, D. y Langslow, A. K. (Eds.) (1999). The Idea of a University. London, Jessica Kingsley Publishers.

Smith, A. \& Webster, F. (Eds.) (1997). The Postmodern University? Contested Visions of Higher Education in Society. Buckingham: SRHE and Open University Press.

Sotelo Martínez, I. (2003). La invención de Europa: la realidad histórico-cultural de Europa. En AA.VV., La formación de europeos. Madrid: Academia Europea de Ciencias y Artes.

Watson, D. (2007). Whatever happened to the Dearing Report? UK higher education 1997-2007. A professional lecture by David Watson. London: Institute of Education.

Watson, D. and Amoah, M. (Eds.) (2007). The Dearing Report. Ten years on. London: Institute of Education, Bedford Way Papers.

Welch, A. (2003). Technocracy, uncertainty and ethics. Comparative Education in an age of postmodernity and globalization. En R. Arnove and C. A. Torres (Eds.), Comparative Education. The Dialectic of the local and the Global. Lanham: Rowman and Littlefield.

Yeatman, A. (1994). Postmodern Revisionings of the Political. London: Routledge.

Young, R. (1997). Comparative methodology and postmodern relativism. International Review of Education, 43(4), 497-505. 